Original article

\title{
Outcome of preterm neonates born to women of a developing country at risk of preterm birth exposed to varying doses of antenatal corticosteroid: A prospective observational study
}

\author{
Supriya, S.N. Singh, Shalini Tripathi*, Mala Kumar \\ Department of Pediatrics, King George's Medical University (KGMU), India
}

\section{A R T I C L E I N F O}

\section{Keywords:}

Antenatal corticosteroid

Developing country

Preterm neonate

Morbidity

Mortality

\begin{abstract}
A B S T R A C T
Background: Antenatal corticosteroid (ACS) is used in women at risk of preterm births to reduce mortality and morbidity of the neonate born. However it's effect may not always be beneficial especially in the women with risk factors for sepsis which is quiet high in developing countries.

Methods: Neonates (26-35 weeks gestational age) delivered to mothers who received 2-4 doses of ACS (group1) or 0-1 dose (group 2) were compared for incidence, severity of Respiratory distress syndrome (RDS) and death. Various morbidities of prematurity were also compared among the two groups.

Results: Less number of neonates developed RDS in group1 $(28.1 \%)$ than group $2(37.8 \%)(p=0.011)$. Few babies needed CPAP in group 1 (34.1\% vs 53.9\%; 0.006), however more babies needed mechanical ventilation (MV) $(62.2 \%$ vs $41.7 \%$; 0.005) in group 1 . Mortality was similar (20.5\% vs $20.1 \%$; $=0.71)$. Group 1 neonates with 2-4 doses of ACS had less Intraventricular hemorrhage (IVH) $(0.3 \%$ vs $2.6 \%$; $p=0.04)$ but sepsis rates ( $12 \%$ vs $6.2 \%$; 0.02$)$ were increased. Other morbidities of prematurity were comparable.

Conclusion: Similar to the previous literature, decrease in incidence of RDS and IVH was found in neonates with higher (2-4) doses of ACS. However more cases of culture positive sepsis and severe RDS occurred in these neonates.
\end{abstract}

\section{Introduction}

Every year, an estimated 15 million babies are born preterm (before 37 completed weeks of gestation), and this number is rising. Across 184 countries, the rate of preterm birth ranges from $5 \%$ to $18 \%$ of babies born. ${ }^{1}$ Preterm birth complications are the leading cause of death among children under 5 years of age, responsible for approximately 1 million deaths in $2015 .^{2}$ In the lower-income countries, on average, $12 \%$ babies are born too early compared with $9 \%$ in higher-income countries. Within countries, poorer families are at higher risk. In top 10 countries with the greatest number of preterm births, India with $3,519,100$ preterm births ranks first. ${ }^{3}$ Preterm neonates are prone to a number of complications. RDS is one of the most early and urgent requiring immediate interventions.

Antenatal corticosteroid (ACS) is an effective therapy to reduce respiratory distress and improve neurological morbidity and mortality in the preterm newborn. ${ }^{4}$ This has become a standard care in cases of imminent or anticipated preterm delivery. ${ }^{5}$ However, a general concern exists regarding the administration of ACS in cases of suspected intrauterine infection. ${ }^{6-8}$ Cochrane database review published in 2006 summarized 21 studies confirmed significant reduction in risk of mortality, Respiratory distress syndrome (RDS) and Intraventricular hemorrhage (IVH). ${ }^{5}$ On the basis of this strong evidence, use of ACS for women at high risk of preterm births is widely recommended by national \& international health organizations. ${ }^{9,10}$ Further studies showed multiple courses do not improve preterm-birth outcomes, and are associated with a decreased weight, length, and head circumference at birth. ${ }^{11}$ Most of the evidence comes from the high income countries with paucity of data from low middle income countries. ${ }^{12}$ Eight studies in 2006 systematic review had increase in puerperal sepsis and fever with dexamethasone. The risks and benefits of ACS in poor population could be very different from those in well nourished, wealthy population with access to round $O$ clock newborn care. A trial done in low and middle income countries including India showed that there was no benefit in preterm infants and rather there was higher neonatal mortality in steroid group. ${ }^{13}$ A possibility is that ACS might compromise a neonate's immune system and increase their susceptibility to infection. ${ }^{13}$ Therefore it is the need of the hour to study the interaction of

\footnotetext{
${ }^{*}$ Corresponding author. Department of Pediatrics, King George's medical university (KGMU), 255/394, Kundri Rakabganj, Lucknow, Uttarpradesh, 226004, India.

E-mail addresses: doctorsupriya0212@gmail.com (Supriya),drsn.singh@rediffmail.com (S.N. Singh), shalini.avasthi@rediffmail.com (S. Tripathi), mala_lko@yahoo.co.in (M. Kumar).
} 
neonatal sepsis with ACS especially in developing countries, hence this study was done.

\section{Methods}

\subsection{Study population}

This study was conducted on consecutively enrolled preterm babies delivered to women at risk of preterm birth. Women delivering preterm babies (between 26 and 35 weeks of gestation) were enrolled and status of ACS coverage was assessed. The preterm infants whose mothers had received 2 or more doses of ACS constituted group 1 and whose mothers either received single dose or not received ACS formed group 2. Neonates with major congenital malformations and suspected congenital heart disease were excluded. Gestational age (GA) was determined based on last menstrual period (LMP) and/or antenatal ultrasonography. Preterm labor diagnosis was based on persistent uterine contraction accompanied by dilatation and or effacement of the cervix.

\subsection{Data collection}

The policy of using ACS included injection dexamethasone, $6 \mathrm{mg}$, intramuscular and repeated $12 \mathrm{~h}$ to maximum of 4 doses. Preterm babies were resuscitated with $\mathrm{T}$ piece resuscitator when required. Preterm babies developing signs of RDS such as presence of at least 2 of the following criteria (NNPD report_2002-03) ${ }^{14}$ : respiratory rare $>60 /$ minute subcostal/intercostal recessions, expiratory grunt/groaning were first initiated on continuous positive airway pressure (CPAP). The criteria for initiation of mechanical ventilation (MV) included any of the following ${ }^{1}$ failure of optimal CPAP ( $>8-9 \mathrm{~cm}$ of water) or apnea on CPAP (ii) Pa02 $<50 \mathrm{mmHg}$ or Sp02 < 89\% on Fi02 0.8 (iii) PaCO2 $>60$ mmHg with pH $<7.25$ (iv) apnea or gasping respiration. The strategy for use of surfactant was early rescue therapy, advised for those who required CPAP with Fi02 40\% or more or on MV. Patients were followed till their outcome. Maternal details like age, parity, socioeconomic status, residence, antenatal care, medical diseases like diabetes, rheumatic heart disease, thyroid disorder, anemia, tuberculosis, HIV infection, pregnancy related disorders: gestational diabetes (GDM), antepartum hemorrhage, pregnancy induced hypertension (PIH), TORCH infection, ACS: doses, timing (period of gestation) route were noted. Intrapartum details like chorioamnionitis (maternal fever, foul smelling discharge, colour of liquor, uterine tenderness, maternal tachycardia, fetal tachycardia, maternal total leukocyte count), meconium stained amniotic fluid, evidence of fetal distress, prolonged labor, obstructed labor, mode of delivery, resuscitation details were noted. Neonatal details: weight, sex, GA, age at onset of symptoms, type of respiratory support required, complications, duration of hospital stay, outcome.

\subsection{Outcomes}

The primary outcomes were recorded in form of occurrence of RDS, its severity and death. Secondary outcomes: occurrence of necrotizing enterocolitis (NEC), sepsis, patent ductus arteriosus (PDA), Intraventricular hemorrhage (IVH), chronic lung disease (CLD), periventricular leukomalacia (PVL) and length of hospital stay were measured. ${ }^{14,15}$

\subsection{RDS diagnosis}

RDS/HMD (Hyaline membrane disease) was diagnosed by presence of all of the three criteria ${ }^{14}$

\section{a. Pre-term neonate}

b. Respiratory distress having onset within $6 \mathrm{~h}$ of birth

c. Amniotic fluid Lecithin/Sphyngomyelin ratio of $<1.5$, or negative gastric aspirate shake test or skiagram of chest showing poor expansion with air bronchogram/reticulogranular pattern/ground glass opacity.
Severity of RDS was decided in accordance with requirement of various form of respiratory support and its duration (oxygen alone, CPAP, MV).

\subsection{Statistical analysis}

Assuming the incidence of RDS in preterm newborn infants with none or single dose of ACS of 30\%, to detect a difference in incidence of RDS of $10 \%$ in preterm babies exposed to 2-4 doses of ACS and accepting two sided alpha error of 5\%, a sample size of 231-293 subjects in each group was needed to give $70-80 \%$ power to the study. Analysis was performed using SPSS soft ware, version 21. Descriptive analysis of qualitative and quantitative data presented as frequency table and mean \pm standard deviation or median (inter-quartile range) respectively. Categorical data was analyzed using Chi-square test or Fisherexact test and continuous data by $t$-test or Mann-Whitney $U$ test. A pvalue of 0.05 was considered to be statistically significant.

\section{Ethical approval}

Ethical clearance was obtained from the Institutional ethical committee.

\section{Results}

Total number of deliveries was 8637 during study period, of which 7860 were live born and 777 were still born. Of 7860 live births, 1273 were preterm births. 677 Neonates were excluded due to congenital malformations, congenital heart defect or refusal to take part in the study. A total of 596 preterms were enrolled and on the basis of maternal exposure to ACS, 292 were grouped in group 1 and 304 in group 2 . Among the 292 neonates in group 1 , who received 2, 3 and 4 doses of ACS were $17(5.8 \%), 46(15.8 \%)$ and $229(78.4 \%)$ respectively. Of 304 neonates in group 2171(56.2\%) received nil dose and 133 (43.8\%) received 1 dose. As shown in Table 1 , the base line neonatal characteristics were similar among two groups. Antenatal maternal characteristics were also comparable except group 1 had more primipara mothers as compared to group2 $(78.1 \%$ vs $69.4 \%$; $=0.02)$.

As shown in Table 2, incidence of RDS in group 1 was $28.1 \%$ and $37.8 \%$ in group 2 , which was statistically significant ( $p=0.011$ ). Severity of RDS was assessed by the need of different respiratory support is shown in Table 2. More RDS babies in group 1(62.2\%) required MV as compared to group $2(41.7 \%)$, and it was statistically significant ( $\mathrm{p}=0.005$ ). Although less number of RDS neonates needed CPAP (34.1\% vs $53.9 \%$; 0.006 ) and oxygen ( $3.7 \%$ vs $4.3 \%$; 0.81 ). $60(20.5 \%)$ babies expired in group 2 as compared to $61(20.1 \%)$ in group 1 which was statistically insignificant $(\mathrm{p}=0.71)$. However among the 60 expired neonates of group $1,35 / 60(58.3 \%)$ were culture positive sepsis verses 19/61(31.1\%) in group 2 ( $\mathrm{p}<0.001)$.

On comparing neonatal morbidities (Table 3) were similar in both the groups except IVH, which was less in group $1(0.3 \%$ vs $2.6 \%$; $\mathrm{p}=0.04)$ and culture positive sepsis which was on the contrary more in group $1(12 \%$ vs $6.2 \%$; $=0.02)$. Occurrence of NEC ( $3.8 \%$ vs $3.6 \%$; $\mathrm{P}=0.93)$, PDA $(7.2 \%$ vs $9.9 \% ; \mathrm{P}=0.25)$, CLD $(2.4 \%$ vs $1.6 \%$; $\mathrm{p}=0.57)$, PVL ( $1 \%$ vs $0.3 \%$; $=0.31)$, and length of stay ( 5 days [3-9.5] vs 5 days [3-10]; $\mathrm{p}=0.43$ ) were comparable.

\section{Discussion}

We studied, effect of 2-4 doses of ACS compared to 0-1 dose between 26 and 34 weeks gestation. We used dexamethasone as ACS, which is also recommended by WHO. A Cochrane review (2013) comparing use of different ACS in women at risk of preterm delivery, found no statistical difference between dexamethasone and betamethasone as far as RDS (RR: $1.06,95 \% \mathrm{Cl} 0.88-1.27$ ), neonatal deaths (RR: 1.41, Cl 0.54-3.67) were concerned. Although there was a 
Table 1

Baseline Maternal \& Neonatal demographic variables.

\begin{tabular}{|c|c|c|c|}
\hline Maternal variables & Group $1(n=292)$ & Group $2(n=304)$ & $P$ value \\
\hline Age (year) mean $\pm S D$ & $25.8 \pm 2.7$ & $26.1 \pm 2.6$ & 0.16 \\
\hline Primipara & $228(78.1 \%)$ & $211(69.4 \%)$ & 0.02 \\
\hline Low Socioeconomic status & $89(30.5 \%)$ & $80(26.3 \%)$ & 0.26 \\
\hline Anemia & $51(17.5 \%)$ & $60(19.7 \%)$ & 0.48 \\
\hline Gestational Diabetes mellitus & $10(3.4 \%)$ & $8(2.6 \%)$ & 0.57 \\
\hline Pregnancy induced hypertension & $89(30.5 \%)$ & $88(28.9 \%)$ & 0.68 \\
\hline Fever $<7$ days of delivery & $5(1.7 \%)$ & $4(1.3 \%)$ & 0.75 \\
\hline Premature rupture of membrane & $95(32.5 \%)$ & $99(32.6 \%)$ & 0.99 \\
\hline Prolonged rupture of membrane & $52(17.8 \%)$ & $59(19.4 \%)$ & 0.62 \\
\hline Urinary tract infection & $2(0.7 \%)$ & $5(1.6 \%)$ & 0.45 \\
\hline Tuberculosis & $1(0.3 \%)$ & $0(0 \%)$ & 0.90 \\
\hline Antenatal Checkup $\geq 3$ & $150(51.4 \%)$ & $141(46.4 \%)$ & 0.25 \\
\hline Hypothyroidism & $17(5.8 \%)$ & $12(3.9 \%)$ & 0.29 \\
\hline Rheumatic Heart Disease & $5(1.7 \%)$ & $9(3.0 \%)$ & 0.42 \\
\hline Chorioamnionitis & $16(5.5 \%)$ & $20(6.6 \%)$ & 0.57 \\
\hline Obstructed Labor/Prolonged Labor & $6(2.0 \%)$ & $7(2.2 \%)$ & 0.81 \\
\hline Meconium Stained Amniotic Fluid & $6(2.1 \%)$ & $15(4.9 \%)$ & 0.06 \\
\hline Abnormal Non Stress Test & $54(38.8 \%)$ & $47(37.9 \%)$ & 0.90 \\
\hline Ante Partum Hemorrhage & $58(19.9 \%)$ & $79(26 \%)$ & 0.08 \\
\hline Neonatal variables & Group $1(n=292)$ & Group $2(n=304)$ & $P$ value \\
\hline Mode of Delivery - Vaginal & $147(49.7 \%)$ & $174(57.2 \%)$ & 0.09 \\
\hline Caesarian Section & $145(49.3 \%)$ & $130(42.8 \%)$ & \\
\hline \multicolumn{4}{|l|}{ Resuscitation } \\
\hline Bag \& Mask & $5(1.7 \%)$ & $6(1.6 \%)$ & 0.73 \\
\hline Intubation with Intermittent Positive Pressure Ventilation & $24(8.2 \%)$ & $33(10.9 \%)$ & \\
\hline Surfactant administration & $28(9.6 \%)$ & $30(9.9 \%)$ & 0.91 \\
\hline Birth weight (gram) mean \pm SD & $1617 \pm 434$ & $1628 \pm 386$ & 0.75 \\
\hline \multicolumn{4}{|l|}{ Weight Category: } \\
\hline$<1000 \mathrm{~g}$ & $27(9.2 \%)$ & $15(4.9 \%)$ & \\
\hline $1000-1499 \mathrm{~g}$ & $101(34.6 \%)$ & $109(35.9 \%)$ & 0.21 \\
\hline$\geq 1500 \mathrm{~g}$ & $164(56.2 \%)$ & $200(59.2 \%)$ & \\
\hline Gestational age (weeks) mean \pm SD & $31.7 \pm 1.8$ & $31.9 \pm 1.6$ & 0.11 \\
\hline
\end{tabular}

$\mathrm{SD}=$ Standard Deviation

Table 2

Incidence and Severity of RDS \& Mortality among the two groups.

\begin{tabular}{llll}
\hline Parameter & Group 1 $(\mathrm{n}=292)$ & Group 2 $(\mathrm{n}=304)$ & P value \\
\hline $\begin{array}{l}\text { Incidence of RDS } \\
\text { Severity of RDS }\end{array}$ & $82(28.1 \%)$ & $115(37.8 \%)$ & 0.011 \\
$\mathrm{O}_{2}$ alone & $3 / 82(3.7 \%)$ & $5 / 115(4.3 \%)$ & 0.81 \\
CPAP & $28 / 82(34.1 \%)$ & $62 / 115(53.4 \%)$ & 0.006 \\
Mechanical ventilation & $51 / 82(62.2 \%)$ & $48 / 115(41.7 \%)$ & 0.005 \\
Outcome & & & \\
Mortality & $60(20.5 \%)$ & $61(20.1 \%)$ & 0.71 \\
LAMA & $3(1.0 \%)$ & $6(2.0 \%)$ & \\
\hline
\end{tabular}

RDS $=$ Respiratory Distress Syndrome, CPAP $=$ Continuous Positive Airway Pressure.

LAMA = Left Against Medical Advice.

statistical reduction in IVH with dexamethasone use, there was no significant difference in severity of IVH and PVL. ${ }^{16}$ In an experimental model in lamb, the minimal interval from fetal exposure to corticosteroid till delivery for improved postnatal lung function was found between 8 and 15 h. ${ }^{17}$ The maximum interval reported was $24 \mathrm{~h}$ to 7 days after dose completion, when the reduction in RDS risk was nearly $50 \% .{ }^{18}$ In the present study, the patients in group 2 received $0-1$ dose of ACS and delivered within $12 \mathrm{~h}$, expecting no benefits from a single dose of ACS. The efficacy of ACS treatment depends on GA. Recent Cochrane database does not support its use in term babies delivered by cesarean. ${ }^{19}$ Administration of ACS before 22 weeks of gestation is unlikely to improve lung function, because of very few primitive alveoli at this GA on which drug can exert its effect. A 2016 meta-analysis of RCTs of ACS before 24 weeks of gestation demonstrated a reduction in perinatal mortality at 23 weeks of gestation, however no statistical reduction was observed for RDS, severe IVH or $\mathrm{NEC}^{20}$ In an
Table 3

Morbidities among the two groups.

\begin{tabular}{llll}
\hline & Group 1 (n=292) & Group 2 (n=304) & P value \\
\hline NEC & $11(3.8 \%)$ & $11(3.6 \%)$ & 0.93 \\
PDA & $21(7.2 \%)$ & $30(9.9 \%)$ & 0.25 \\
IVH Grade 3 (at least) & $1(0.3 \%)$ & $8(2.6 \%)$ & 0.04 \\
PVL & $3(1.0 \%)$ & $1(0.3 \%)$ & 0.31 \\
CLD & $7(2.4 \%)$ & $5(1.6 \%)$ & 0.57 \\
Positive Sepsis Screen & $61(20.9 \%)$ & $83(27.3 \%)$ & $\mathbf{0 . 0 2}$ \\
Positive Blood Culture & $35(12.0 \%)$ & $19(6.2 \%)$ & \\
Hospital Stay (Days) & $5(3-9.5)$ & $5(3-10)$ & 0.43 \\
$\quad$ & & \\
\hline
\end{tabular}

$\mathrm{NEC}=$ Necrotising Enterocolitis, $\mathrm{PDA}=$ Patent Ductus Arteriosus, $\mathrm{IVH}=$ Intra Ventricular Hemorrhage.

$\mathrm{PVL}=$ Peri ventricular Leucomalacia, $\mathrm{CLD}=$ Chronic Lung Disease, $\mathrm{IQR}=$ Inter Quartile Range.

observational cohort study on extremely premature infants (birth weight, 401-1000 g; GA 22-27 weeks) logistic regression revealed that complete (odds ratio, 0.63; 95\% CI, 0.53-0.76), partial (odds ratio, 0.77; 95\% CI, 0.63-0.95) ACS courses were associated with lower rates of death or neurodevelopmental impairment compared with no ACS group, mediated by ACS-associated reductions in rates of severe IVH and/or cystic PVL. ${ }^{21}$ We studied women at risk of preterm delivery (26-34 weeks) and preterm babies delivered within the next 7 days. Use of ACS after 34 weeks of gestation is controversial because of inconsistent data about its efficacy, concerns for neonatal hypoglycemia and long term safety. In a triple blinded RCT by Porto et al. no reduction in respiratory disorders of newborns was found. ${ }^{22}$ However a multicentric randomized controlled trial (RCT) on 1427 late preterm neonates (2016) found significant reduction in neonatal respiratory mortality, morbidity. ${ }^{23}$ Authorities like American college of obstetrician and 
gynecologist, Royal College of obstetrician and gynecologist have recommended it's use beyond 34 weeks of gestation. , $10^{2}$

In our study, the incidence of RDS was $9.7 \%$ lesser in group I babies (2-4 doses of ACS) (28.1\% vs $37.8 \%)$ compared to group 2 (0-1 dose of ACS) which is statistically significant $(\mathrm{p}=0.011)$. The results are similar to cochrane review (2006), which found decreased risk of RDS by $31 \%(95 \%$ CI $27-41 \%$, 4038 infants) in babies who were steroid covered (betamethasone, dexamethasone or hydrocortisone). ${ }^{5} \mathrm{~A}$ project in four South East Asian countries, (SEA-ORCHID, 2008) found that infants exposed to ACS were less likely to be stillborn (RR 0.06, [95\%CI 0.02, $0.25], \mathrm{p}=0.01$ ) and were less likely to die prior to hospital discharge (stillbirth and death prior to discharge combined, RR 0.49, [95\%CI $0.30,0.79], \mathrm{p}=0.01$ ) compared with infants who were not exposed (31). Roberto et al. from Brazil (2016) found similar incidence of RDS in both the groups, but their baseline maternal and neonatal parameters were not comparable. ${ }^{24}$

We found severity of RDS was more in group 1 as $62.2 \%$ babies required MV compared to $41.7 \%$ in group 2 . That is although less babies developed RDS but those who developed were more severe than less or unexposed group. Hiroshi Ishikawa (2015) showed that ACS does not affect short- or long-term outcome in SGA infants when birthweight is less than $1500 \mathrm{~g} .{ }^{25}$ We have equal proportion of SGA babies in the two groups. Besides, there are differences in the genetic makeup, ethnicity, and nutritional status of mothers in our country which might influence the incidence \& severity of RDS.

The baseline characteristics were comparable except caesarean sections were more in group 1 babies (49.3\% Vs $42.8 \%$; 0.09). This could have contributed to increased severity of RDS in group I patients. In a study done in middle-low income developing countries by Fernando Althabe it was seen that despite increased use of ACS in lowbirth weight infants in the intervention group, neonatal mortality did not decrease in this group and increased in the population overall $(27.4 / 1000$ vs $23.9 / 1000, \quad R R=1.12,95 \%$ CI $1.02-1.22$, pvalue $=0.0127$ ). For every 1000 women exposed to this strategy, an excess of 3.5 neonatal deaths occurred, and the risk of maternal infection seems to have been increased. ${ }^{13}$ In a recent study ACS use decreased oxygen dependency at 36 weeks in $11 \%$, advanced resuscitation in delivery room in $24 \%$, severe IVH in $12 \%$, PDA requiring surgery in $3.6 \%$ and retinopathy of prematurity in $0.3 \%$, but increased the probability of late-onset sepsis in $2.5 \%{ }^{26}$ Similar to these two studies, we had more cases of culture positive sepsis occurred in group1.

We found mortality was similar in both the groups $(20.5 \% \mathrm{Vs}$ 20.1\%; p value-0.71). Similar results were depicted by Onland \& colleagues with no significant reduction in neonatal morbidity and mortality with steroid use. ${ }^{27}$ A Cochrane review (18 RCT) found ACS was associated with reductions in neonatal mortality in very preterm babies $(\mathrm{RR}=0.66 ; 95 \% \mathrm{Cl} 0.59-0.73 ; 21$ studies, 4038 babies $) .{ }^{28}$ Contrary to this there was no effect of ACS on mortality in our study. However all the trials included in the cochrane review were from developed country. Elimian A (2003) like us studied 74 women exposed to ACS \& 221 women not exposed to ACS and found no statistically significant difference in the rate of any adverse neonatal morbidity $(47.3 \%$ vs. $40.7 \%, \mathrm{p}=0.32)$ or the rate of neonatal morbidity composite $(34.4 \%$ vs. $37.8 \% \mathrm{p}=0.59$ ) between the two groups. ${ }^{29} \mathrm{~A}$ cochrane review (2006) of 21 studies (3885 women and 4269 infants) showed that treatment of women at risk of preterm birth with a single course of ACS reduced the risk of neonatal death by $31 \%$ (95\% CI 19-42\%), RDS by $44 \%$ (95\% Cl 31-57\%) and IVH by $46 \%$ (95\% Cl 31\%-67\%)..$^{5}$ ACS use reduced neonatal deaths even when infants were born less than $24 \mathrm{~h}$ after the first dose (RR 0.53, 95\% CI 0.29-0.96, four studies, 295 infants) till 7 days. ${ }^{5}$ In our study we found reduction in the incidence of IVH $(0.3 \%$ vs $2.6 \% ; \mathrm{p}=0.04)$ but other morbidities like NEC $(3.8 \%$ vs $3.6 \%$; $\mathrm{P}=0.93)$, PDA (7.2\% vs $9.9 \%$; $\mathrm{P}=0.25)$, CLD $(2.4 \%$ vs $1.6 \%$; $\mathrm{p}=0.57)$, PVL ( $1 \%$ vs $0.3 \% ; \mathrm{P}=0.31)$, and length of stay (5 days [3-9.5] vs 5 days [3-10]; $\mathrm{p}=0.43$ ) remained same.

In our study the incidence of maternal infection was similar in 2 groups, assessed by evidence of clinical chorioamnionitis $(5.5 \%$ vs $6.6 \%$; 0.57$)$, puerperal sepsis ( $0.4 \%$ vs nil; 0.28$)$ and use of antibiotics in the mothers $(13.6 \%$ vs $15.7 \%$; 0.50$)$. According to cochrane review (2006) there was no increased risk of chorioamnionitis, puerperal sepsis in mother receiving ACS. Similar results were found in a recent cochrane review (2016) showing no significant association between a single course of ACS and chorioamnionitis (RR-0.91,95\% Cl,0.7-1.18, 12 studies, 2485 women) or puerperal sepsis. ${ }^{28}$ Although a study (F Althabe 2014), from developing country found increased incidence in maternal infections ( $3 \%$ vs $2 \%$, p-value- $<0.0001$ ) with ACS. ${ }^{13}$ This may be because this study was done in developing low income countries verses the earlier studies were from developed world.

\subsection{Strengths and limitations of the study}

The study was well planned and conducted however the study was single centered and because of the small sample size the power of the study was less than $80 \%$ so subgroup analysis was limited by small number of patients.

\subsection{Interpretation}

The present study demonstrated marginal decrease in the incidence of RDS and IVH in the preterm babies whose mothers received 2-4 doses of ACS compared to those with 0-1 dose. However there was trend to have more cases of severe RDS and higher sepsis among neonates with the higher dose group. Also because of resource poor setting surfactant and CPAP was not available to many of the babies where it was indicated, which would have changed the outcome of these patients.

\section{Conclusion}

Similar to the previous studies, our study too shows reduction in neonatal RDS and IVH cases by ACS however the substantial increase in the neonatal sepsis rates with more severe RDS in the ACS group, elucidates this issue being sprouted by few earlier studies especially in low middle income country like ours.

\section{Funding}

There was no funding for this study.

\section{Declaration of competing interest}

There is no conflict of interest with respect to research, authorship, and publication of this article.

\section{References}

1. World health organization. Preterm Birth. 19.2. 2018; 2018. Available from: https:// www.who.int/news-room/fact-sheets/detail/preterm-birth.

2. Liu L, Oza S, Hogan D, et al. Global, regional, and national causes of under-5 mortality in 2000-15: an updated systematic analysis with implications for the Sustainable Development Goals. Lancet. 2016;388(10063):3027-3035.

3. Blencowe H, Cousens S, Oestergaard M, et al. National, regional and worldwide estimates of preterm birth. Lancet. June 2012;379(9832):2162-2172 9.

4. Mwansa-Kambafwile J, Cousens S, Hansen T, Lawn JE. Antenatal steroids in preterm labor for the prevention of neonatal deaths due to complication of preterm birth. Int $J$ Epidemiol. 2010;39(Suppl 1):i122-i133.

5. Roberts D, Dalziel S. Antenatal corticosteroids for accelerating fetal lung maturation for women at risk of preterm birth. Cochrane Database Syst Rev. 2006 Jul 19(3):CD004454

6. Goldenberg RL, Andrews WW, Faye-Petersen OM, Cliver SP, Goepfert AR, Hauth JC. The Alabama preterm birth study: corticosteroids and neonatal outcomes in 23 to 32 week newborns with various markers of intrauterine infection. Am J Obstet Gynecol 2006;195:1020-1024

7. Been JV, Degraeuwe PL, Kramer BW, Zimmermann LJ. Antenatal steroids and neonatal outcome after chorioamnionitis: a meta-analysis. BJOG. 2011 Jan;118(2):113-122. 
8. Wu YW. Systematic Review of chorioamnionitis and cerebral palsy. Ment Retard Dev Disabil Res Rev. 2002;8:25-29.

9. Royal College of Obstetricians and gynaecologists. In: RCOG Green - Top Guideline No. 7. Antenatal Corticosteroids to Reduce Neonatal Morbidity and Mortality. vol. 7. London: RCOG; 2010.

10. Antenatal corticosteroid therapy for fetal maturation. . Available from:- https:// www.acog.org/Clinical-Guidance-and-Publications/Committee-Opinions/ Committee-on-Obstetric-Practice/Antenatal-Corticosteroid-Therapy-for-FetalMaturation.

11. Murphy KE, Hannah ME, Willan AR, et al. MACS Collaborative Group. Multiple courses of antenatal corticosteroids for preterm birth (MACS): a randomised controlled trial. Lancet. 2008 Dec 20;372(9656):2143-2151. https://doi.org/10.1016/ S0140-6736(08)61929-7.

12. Azad K, Costello A. Extreme caution is needed before scale up of antenatal corticosteroids to reduce preterm deaths in low income setting. Lancet Glob Health. 2014;2:e191-e192.

13. Althabe F, Belizan JM, McClure Em, Hemingway-Foday J, Berrueta M, etal Mazzoni A. A population-based, multifaceted strategy to implement antenatal corticosteroid treatment versus standard care for the reduction of neonatal mortality due to preterm birth to low-income and middle-income countries the ACT cluster randomized trial. The Lancet. 2015;385:629-639.

14. National neonatal perinatal database. Human reproduction research centre network, report 2002-2003, supported by Indian council of medical research New Delhi. Available from: http://newbornwhocc.org/pdf/HRRC-Report_2002-03.pdf.

15. Agarwal R, Deorari A, Paul V, Sankar MJ, Sachdeva A. AIIMS Protocols in Neonatology. second ed. New Delhi: Noble publisher; 2019.

16. Brownfoot FC, Gagliardi DI, Bain E, et al. Different corticosteroids and regimens for accelerating fetal lung maturation for women at risk of preterm birth. Cochrane Database Syst Rev. 2013(8) CD006764.

17. Ilkegame M, Jobe Polk D. Mimimum interval form fetal betamethasone treatment to postnatal lung responses in preterm lambs. Am J Obstet Gynecol. $1996 ; 174: 1408-1413$

18. Robert D, Dalziel SR. Antenatal Corticosteroids for Accelerating Fetal Lung Maturation for Women at Risk of Preterm Birth Cochrane Database Syst Rev. 2016; 2016 CD004454.

19. Sotiriadis A, Makrydimas G, Papatheodorou S, Ioannidis JP, McGoldrick E.
Corticosteroids for preventing neonatal respiratory morbidity after elective caesarean section at term. Cochrane Database Syst Rev. 2018 Aug 3;8. https://doi.org/10. 1002/14651858.CD006614 CD006614.

20. Park CK, Isayama T, McDonald SD. Antenatal corticosteroids therapy before 24 Weeks of gestation: a systematic review and meta-analysis. Obstetr Gynecol. 2016;127:715-725.

21. Alexander N, Rosenlocher F, Dettenborn L, et al. Impact of antenatal glucocorticoid therapy and risk of preterm delivery on intelligence in term-born children. J Clin Rndocrinol Metab. 2016 Feb;101(2):581-589.

22. Porto AMF, Coutinho IC, Correia JB, Amorim MMR. Effectiveness of antenatal corticosteroids in reducing respiratory disorders in late preterm infants:randomised clinical trial. BMJ. 2011;342:d1696.

23. Gyamfi-Bannerman C, Thom EA, Blackwell SC, Tita AT, Reddy UM, GRet al Saade. Antenatal betamethasone for women at risk for late preterm delivery. $N$ Engl J Med. 2016 Apr 7;374(14):1311-1320.

24. Pattanittum P, Ewens MR, Laopaiboon M, Lumbiganon P, McDonald SJ, Crowther CA. The SEA-ORCHID Study Group. Use of antenatal corticosteroids prior to preterm birth in four South East Asian countries within the SEA-ORCHID project. BMC Pregnancy Childbirth. 2008;8:47.

25. Ishikawa Hiroshi, Miyazaki Ken, Ikeda Tomoaki, Murabayashi Nao, Hayashi Kazutoshi. Akihiko kai the effects of antenatal corticosteroids on short and long term outcomes in small for-gestational-age infants. Int J Med Sci. 2015;12(4):295-300.

26. Ogata Fonseca MCM, Almeida MFB, Guinsburg R. Antenatal corticosteroids: analytical decision model and economic analysis in a Brazilian cohort of preterm infants. $J$ Matern Fetal Neonatal Med. 2016;29(18)

27. Onland W, De Laat MW, etal Mol BW. Effects of antenatal corticosteroids given prior to 26' weeks gestation; a systematic review of randomized controlled trials. Am J Perinant. 2011;28:33-44

28. Roberts D, Brown J, Medley N, Dalziel SR. Antenatal corticosteroids for accelerating fetal lung maturation for women at risk of preterm birth. Cochrane Database Syst Rev. 2016 Mar 21;3 CD004454.

29. Elimian A, Figueroa R, Spitzer AR, Ogburn PL, Wieneck V, Quirk JG. Antenatal corticosteroid are incomplete courses beneficial? Am Coll Ostet Gynaecol. August, 2003;102(2). 